\title{
SOME BANACH ALGEBRAS WITHOUT DISCONTINUOUS DERIVATIONS
}

\author{
BRIAN FORREST
}

(Communicated by J. Marshall Ash)

\begin{abstract}
It is shown that the completion of $A(G)$ in either the multiplier norm or the completely bounded multiplier norm is a Banach algebra without discontinuous derivations when $G$ is either $F_{2}$ or $\operatorname{SL}(2, \mathbb{R})$.
\end{abstract}

\section{INTRODUCTION}

In [6], it was shown that a locally compact group $G$ is amenable if and only if every derivation $D$ from $A(G)$, the Fourier algebra of $G$, into an arbitrary Banach $A(G)$-bimodule $X$ is continuous. The same result can be shown to hold for the Herz algebras $\operatorname{Ap}(G), 1<p<\infty$ [7]. Since the class of amenable groups is substantial (it includes all compact groups and all commutative groups) a large number of Banach algebras without discontinuous derivations have been identified. However, since $\operatorname{SL}(2, \mathbb{R})$ and $F_{2}$, the free group on two generators, are nonamenable, $A(\operatorname{SL}(2, \mathbb{R}))$ and $A\left(F_{2}\right)$ have discontinuous derivations. We will show that both $A(\operatorname{SL}(2, \mathbb{R}))$ and $A\left(F_{2}\right)$ can be given natural norms in such a way that their completions will be Banach algebras without discontinuous derivations.

\section{PReliminaries AND NOtATION}

Let $G$ be a locally compact group. Let $A(G)$ be the Fourier algebra of $G$ as defined by P. Eymard in [4]. $A(G)$ is a Banach algebra with respect to pointwise multiplication. It is also the predual of the von Neumann algebra $\mathrm{VN}(G)$ associated with the left-regular representation of $G$ on $L^{2}(G)$ and a closed ideal in $B(G)$, the Fourier Stieltjes algebra of $G$.

Let $M A(G)$ denote the space of multipliers of $A(G)$. That is, the continuous functions $\psi$ on $G$ such that $\psi u \in A(G)$ for every $u \in A(G)$. For each $\psi \in M A(G), u \in A(G)$, let $m_{\psi}(u)=\psi u$. Denote by $\|\psi\|_{m}$ the operator norm of $m_{\psi}$. We call $\psi$ a completely bounded multiplier of $A(G)$ if $m_{\psi}^{*}$, the adjoint of $m_{\psi}$, is a completely bounded map on $\operatorname{VN}(G)$ [3]. Let $\|\psi\|_{M_{0}}$ be the

Received by the editors May 30, 1990 and, in revised form, September 4, 1990.

1980 Mathematics Subject Classification (1985 Revision). Primary 43A07, 43A15; Secondary $46 \mathrm{~J} 10$.

Key words and phrases. Fourier algebra, multipliers, completely bounded multiplier, derivation, free group. 
completely bounded norm of $m_{\psi}^{*}$ and let $M_{0} A(G)$ denote the Banach algebra of completely bounded multipliers of $A(G)$. Then $B(G) \subseteq M_{0} A(G) \subseteq M A(G)$ and $\|u\|_{M} \leq\|u\|_{M_{0}} \leq\|u\|_{B(G)}$ for every $u \in B(G)$.

We will denote by $A_{M}(G)$ and $A_{M_{0}}(G)$ the closure of $A(G)$ in $M A(G)$ and in $M_{0} A(G)$ respectively. It is well known that $G$ is amenable if and only if $M A(G)=B(G)[12,13]$. In this case $\|u\|_{B(G)}=\|u\|_{M}$ for every $u \in B(G)$ and hence $A(G)=A_{M}(G)=A_{M_{0}(G)}$.

If $\mathscr{A}$ is a Banach algebra, then a derivation $D$ on $\mathscr{A}$ is a linear map from $\mathscr{A}$ into a Banach $\mathscr{A}$-bimodule $X$ such that $D(u v)=u \cdot D(v)+D(u) \cdot v$ for every $u, v \in \mathscr{A}$. If $\mathscr{A}$ is commutative, we will denote the maximal ideal space of $\mathscr{A}$ by $\Delta(\mathscr{A})$. We also use $\Delta(\mathscr{A})$ to denote the multiplicative linear functionals which correspond to the maximal ideal. Given a closed subset $A$ of $\Delta(\mathscr{A})$, we denote by $I_{\mathscr{A}}(A)$, the ideal $\{u \in \mathscr{A} ; u(x)=0$ for every $x \in A\}$, where $\mathscr{A}$ is realized as an algebra of functions on $\Delta(\mathscr{A})$ by means of the Gelfand transform. $A$ is called a set of spectral synthesis for $\mathscr{A}$ or simply an $S$-set if $I_{\mathscr{A}}^{0}(A)=\{u \in \mathscr{A} ; \operatorname{supp} u$ is compact, supp $u \cap A=\varnothing\}$ is dense in $I_{\mathscr{A}}(A)$.

\section{SPECTRAL SYNTHESIS AND AUTOMATIC CONTINUITY OF DERIVATIONS}

Lemma 1. Let $G$ be a locally compact group. Then

$$
\Delta\left(A_{M}(G)\right)=\Delta\left(A_{M_{0}}(G)\right)=G .
$$

Proof. Let $\psi \in \Delta\left(A_{M}(G)\right)$. Then $\left.\psi\right|_{A(G)}$, the restriction of $\psi$ to $A(G)$, belongs to $\Delta(A(G))$. By [4, p. 222] there exists an $x \in G$ such that $\psi(u)=u(x)$ for every $u \in A(G)$.

Let $v \in A_{M}(G)$. As $A(G)$ is dense in $A_{M}(G)$, we can find $\left\{u_{k}\right\} \subseteq A(G)$ such that $\left\|u_{k}-v\right\|_{M} \rightarrow 0$. However, convergence in $A_{M}(G)$ implies convergence in the topology of uniform convergence on compacta and hence in the topology of pointwise convergence. Therefore $\psi(v)=\lim _{k} \psi\left(u_{k}\right)=\lim _{k} u_{k}(x)=v(x)$. It follows that as a set $\Delta\left(A_{M}(G)\right)=G$.

Now suppose that $\left\{x_{\alpha}\right\}_{\alpha \in I}$ is a net in $G$ such that $x_{\alpha}$ converges to $x \in G$ in the usual topology on $G$. Then for each $u \in A_{M}(G), u\left(x_{\alpha}\right) \rightarrow u(x)$. Hence $x_{\alpha} \rightarrow x$ in the $\sigma\left(A_{M}(G)^{*}, A_{M}(G)\right)$ topology. Conversely, if $x_{\alpha} \rightarrow x$ in the $\sigma\left(A_{M}(G)^{*}, A_{M}(G)\right)$ topology, then for every $u \in A(G), u\left(x_{\alpha}\right) \rightarrow u(x)$. Since $G \subseteq A(G)^{*}, x_{\alpha} \rightarrow x$ in the $\sigma\left(A_{M}(G)^{*}, A_{M}(G)\right)$ topology. But $\Delta(A(G))$ is homeomorphic to $G$ so $x_{\alpha} \rightarrow x$ in $G$. Therefore the $\sigma\left(A_{M}(G)^{*}, A_{M}(G)\right)$ topology agrees with the usual topology on $G$.

A similar argument establishes that $\Delta\left(A_{M_{0}}(G)\right)=G$.

Proposition 1. Let $\mathscr{A}$ be either $A_{M}(G)$ or $A_{M_{0}}(G)$. Then $\{e\}$ is an $S$-set of $\mathscr{A}$.

Proof. Let $v \in \mathscr{A}$ with $v \in I_{\mathscr{A}}(\{e\})$. Then there exists $u_{k} \in A(G)$ with $\left\|u_{k}-v\right\|_{\mathscr{A}} \rightarrow 0$. Furthermore since $u_{k}(e) \rightarrow v(e)=0$, we can assume that $\left|u_{k}(e)\right|<1 / 2 k$. We can also find $w_{k} \in A(G)$ with $\left\|w_{k}\right\|_{A(G)}=w_{k}(e)=u_{k}(e)$. Let $v_{k}=u_{k}-w_{k}$. Then $v_{k}(e)=0$. Since $v_{k} \in I_{A(G)}(\{e\})$ and $\{e\}$ is an $S$-set for $A(G)$ [4, p. 229], there exists $z_{k} \in A(G)$ with $\left\|z_{k}-v_{k}\right\|_{A(G)} \leq 1 / 2 k$, 
$\operatorname{supp} z_{k}$ is compact and $\operatorname{supp} z_{k} \cap\{e\}=\varnothing$. However

$$
\begin{aligned}
\left\|z_{k}-v\right\|_{\mathscr{A}} & \leq\left\|z_{k}-v_{k}\right\|_{\mathscr{A}}+\left\|v_{k}-v\right\|_{\mathscr{A}} \\
& \leq\left\|z_{k}-v_{k}\right\|_{A(G)}+\left\|u_{k}-v\right\|_{\mathscr{A}}+\left\|w_{k}\right\|_{A(G)} \\
& \leq\left\|u_{k}-v\right\|_{\mathscr{A}}+1 / k .
\end{aligned}
$$

Hence $\left\|z_{k}-v\right\|_{\mathscr{A}} \rightarrow 0$. Therefore $I_{\mathscr{A}}^{0}(\{e\})$ is dense in $I_{A}(\{e\})$ and $\{e\}$ is an $S$-set.

Proposition 2. Let $\mathscr{A}$ be either $A_{M}(G)$ or $A_{M_{0}}(G)$. If $\mathscr{A}$ has a bounded approximate identity, then so does $I_{\mathscr{A}}(\{e\})$.

Proof. Let $\left\{u_{\alpha}\right\}_{\alpha \in I}$ be a bounded approximate identity in $\mathscr{A}$. Let $\mathscr{F}(\{e\})=$ $\{K \subset G ; K$ is compact, $K \cap\{e\}=\varnothing\}$. For every $K \in \mathscr{F}(\{e\})$, there exists $v_{K} \in B(G)$ such that $\left\|v_{K}\right\|_{\mathscr{A}} \leq\left\|v_{K}\right\|_{B(G)}=1, u_{K}(e)=1$, and $v_{K}(x)=0$ for every $x \in K$. Define $w_{K, \alpha} \in \mathscr{A}$ by $w_{K, \alpha}=u_{\alpha}-v_{K} u_{\alpha}$. Then $w_{K, \alpha}(e)=0$, $\left\|w_{K, \alpha}\right\|_{\mathscr{A}} \leq 2\left\|u_{\alpha}\right\|_{\mathscr{A}}$, and $w_{K, \alpha} v=u_{\alpha} v$ for every $v \in \mathscr{A}$ with $\operatorname{supp} v \subset K$.

Order $K \times I$ by $\left(K_{1}, \alpha_{1}\right) \leq\left(K_{2}, \alpha_{2}\right)$ if and only if $K_{1} \subseteq K_{2}$ and $\alpha_{1} \leq \alpha_{2}$. If $v \in \mathscr{A}$ and $\operatorname{supp} v \in \mathscr{F}(\{e\})$, then $v=\lim _{K, \alpha} w_{K, \alpha} v$. By Proposition 1, such $v$ 's are dense in $I_{\mathscr{A}}(\{e\})$. As $\left\{w_{K, \alpha}\right\}_{K \times I}$ is bounded, $\lim _{K, \alpha} w_{K, \alpha} w=w$ for every $w \in I_{\mathscr{A}}(\{e\})$.

The proof of Proposition 2 is a modification of the proof of [7, Proposition 3.2]. The case where $G$ is amenable is due to A. Lau [11, Corollary 4.11].

Corollary 1. Let $\mathscr{A}$ be either $A_{M}(G)$ or $A_{M_{0}}(G)$. If $\mathscr{A}$ has a bounded approximate identity, then $\mathscr{A}$ satisfies Ditkin's condition. Furthermore, if $A$ has a closed subset of $G$ and the boundary of $A$ contains no nontrivial perfect set, then $A$ is an $S$-set. In particular, every finite subset of $G$ is an $S$-set for $\mathscr{A}$.

Proof. Let $x \in G$ and $u \in \mathscr{A}$ be such that $u(x)=0$. From the proof of Proposition 2 (translate is $x \neq e$ ), we see there exists a sequence $\left\{v_{n}\right\} \subset \mathscr{A}$ for which each $v_{n}$ vanishes in a neighborhood $V_{n}$ of $\{x\}$ and $\lim _{n}\left\|v_{n} u-u\right\|_{\mathscr{A}}=0$.

Assume that $G$ is not compact. Let $u \in \mathscr{A}, u \neq 0$. Let $\varepsilon>0$. If $\left\{u_{\alpha}\right\}_{\alpha \in I}$ is a bounded approximate identity in $\mathscr{A}$, then there exists $u_{\alpha_{\varepsilon}}$ such that $\left\|u_{\alpha_{\varepsilon}} u-u\right\|_{\mathscr{A}}<\varepsilon / 2$. We can find $v \in A(G)$ with supp $v$ compact such that $\left\|u_{\alpha_{\varepsilon}}-v\right\|_{\mathscr{A}} \leq \varepsilon / 2\|u\|_{\mathscr{A}}$. Then $\|v u-u\|_{\mathscr{A}} \leq\left\|v u-u_{\alpha} u\right\|_{\mathscr{A}}+\left\|u_{\alpha} u-u\right\|_{\mathscr{A}} \leq \varepsilon$.

Hence $\mathscr{A}$ satisfies Ditkin's condition [9, p. 49]. The remaining statements follow immediately from Ditkin's theorem [9, p. 497].

Corollary 1 is simply [7, Lemma 5.2, Proposition 5.3] when $G$ is assumed to be amenable.

Proposition 3. Let $\mathscr{A}$ be either $A_{M}(G)$ or $A_{M_{0}}(G)$. If $\mathscr{A}$ has a bounded approximate identity and $I$ is a closed cofinite ideal in $\mathscr{A}$ (i.e., $\operatorname{dim} \mathscr{A} / I<\infty)$, then $I=I(A)$ for some finite set $A=\left\{x_{1}, \ldots, x_{n}\right\}$ where $n$ is the codimension of $I$.

Proof. Let $A=Z(I)=\{x \in G ; u(x)=0$ for every $u \in I\}$. Let $n=\operatorname{codim} I$. Assume that $A$ contains $n+1$ distinct elements $\left\{x_{1}, \ldots, x_{n+1}\right\}$. We can find a compact neighborhood $V_{k}$ of each $x_{k}$ such that $V_{j} \cap V_{k}=\varnothing$ if $j \neq k$. We can also find $u_{k} \in A(G)$ such that supp $u_{k} \subseteq V_{k}$ and $u_{k}\left(x_{k}\right)=1$ for $1 \leq k \leq n+1$. But if $\psi: \mathscr{A} \rightarrow \mathscr{A} / I$ is the canonical homomorphism, then $\left\{\psi\left(u_{1}\right), \ldots, \psi\left(u_{n+1}\right)\right\}$ is a linearly independent subset, which is impossible if 
$\operatorname{codim} I=n$. Therefore $A$ has at most $n$ elements. Since $A$ is finite, it is an $S$-set by Corollary 1 . Therefore $I=I(A)$.

Let $A=\left\{x_{1}, \ldots, x_{k}\right\}$. Let $u_{k} \in A(G)$ be such that $u_{k}\left(x_{k}\right)=1$ and $u_{k}\left(x_{j}\right)=0$ if $j \neq k$. Let $u \in \mathscr{A}$. Then

$$
u=\sum_{i=1}^{k} u\left(x_{i}\right) u_{i}+\left(u-\sum_{i=1}^{k} u\left(x_{i}\right) u_{i}\right)
$$

Since

$$
u-\sum_{i=1}^{k} u\left(x_{i}\right) u_{i} \in I(A), \quad k \geq n
$$

Hence $k=n$.

Proposition 4. Let $\mathscr{A}$ be either $A_{M}(G)$ or $A_{M_{0}}(G)$. Assume that $\mathscr{A}$ has a bounded approximate identity. Let $I$ be a closed cofinite ideal of $\mathscr{A}$. Then I has a bounded approximate identity. In particular $I^{2}=\left\{\sum_{i=1}^{n} u_{i} v_{i}, u_{i}, v_{i} \in\right.$ $I\}=I$.

Proof. By Proposition 3, $I=I\left(\left\{x_{1}, \ldots, x_{n}\right\}\right)$ for some finite subset of $G$.

Let $A, B$ be two closed subsets of $G$. Assume that $I_{\mathscr{A}}(A)$ and $I_{\mathscr{A}}(B)$ have bounded approximate identities $\left\{u_{i}\right\}_{i \in T},\left\{v_{j}\right\}_{j \in J}$ respectively. Then it is easy to see that $\left\{u_{i} v_{j}\right\}_{T \times J}$ is a bounded approximate identity for $I(A \cup B)$.

Since $\mathscr{A}$ has a bounded approximate identity, Proposition 2 implies that $I_{\mathscr{A}}(\{e\})$ has a bounded approximate identity. By translating, we see that $I_{\mathscr{A}}(\{x\})$ has a bounded approximate identity for every $x \in G$. A simple induction argument shows that $I$ also has a bounded approximate identity. Then $I^{2}=I$ follows from Cohen's factorization theorem [9, p. 268].

Theorem 1. Let $\mathscr{A}$ be either $A_{M}(G)$ or $A_{M_{0}}(G)$. If $\mathscr{A}$ has a bounded approximate identity, then $I$ is a cofinite ideal of $\mathscr{A}$ if and only if $I=I(A)$ for some finite subset $A$ of $G$.

Proof. Proposition 4 shows that every closed cofinite ideal of $\mathscr{A}$ is idempotent. By [2, Theorem 2.3] every cofinite ideal of $\mathscr{A}$ must be closed and is therefore of the form $I(A)$ for some finite subset $A$ of $G$ by Proposition 3 .

If $I=I(A)$ for a finite subset $A$ of $G$, then the proof of Proposition 3 shows that $I$ is cofinite.

If $G$ is amenable, then Proposition 3 and Theorem 1 follow from [7, Corollary 5.6, Theorem 5.8].

Theorem 2. Let $\mathscr{A}$ be either $A_{M}(G)$ or $A_{M_{0}}(G)$. If $\mathscr{A}$ has a bounded approximate identity, then every homomorphism from $\mathscr{A}$ with finite-dimensional range is continuous.

Proof. The statement follows immediately from Theorem 1, Proposition 4, and [2, Theorem 2.3].

Lemma 2. Let $\mathscr{A}$ be either $A_{M}(G)$ or $A_{M_{0}}(G)$. Assume that $\mathscr{A}$ has a bounded approximate identity. Let $I$ be a closed ideal in $\mathscr{A}$ with infinite codimension. Then there exist sequences $\left\{u_{n}\right\},\left\{v_{n}\right\}$ in $A(G)$ such that $u_{n} v_{1} \cdots v_{n-1} \notin I$ but $u_{n} v_{1} \cdots v_{n} \in I$ for $n \geq 2$.

Proof. If $I$ has infinite codimension, then $A=Z(I)=\{x \in G, u(x)=0$ $\forall u \in I\}$ must be infinite by Theorem 1 . 
From the proof of [6, Lemma 2], we see that we can find sequences $\left\{u_{n}\right\}$, $\left\{v_{n}\right\} \subseteq A(G)$ such that $u_{n} v_{1} \cdots v_{n-1} \notin I_{A(G)}(A)$ while $u_{n} v_{1} \cdots v_{n}=0$. Since $I_{A(G)}(A) \subseteq I_{\mathscr{A}}(A)$, the result follows.

Theorem 3. Let $\mathscr{A}$ be either $A_{M}(G)$ or $A_{M_{0}}(G)$. Assume that $\mathscr{A}$ has a bounded approximate identity. Then every derivation $D$ of $\mathscr{A}$ into a Banach $\mathscr{A}$-bimodule $X$ is continuous.

Proof. This follows immediately from Proposition 4, Lemma 2, and [10, Theorem 2].

For the algebra $A(G)$, the automatic continuity properties listed in Theorems 2, 3 are characteristic of amenable groups. We see that if $A_{M}(G)$ or $A_{M_{0}}(G)$ has a bounded approximate identity, then it possesses many of the properties of the Fourier algebra of an amenable group.

In [5] it was shown that $A\left(F_{2}\right)$ has an approximate identity which is necessarily unbounded in $A(G)$ but is bounded in $\|\cdot\|_{M}$. Hence for the prototypical nonamenable group $F_{2}, A_{M}\left(F_{2}\right)$ has a bounded approximate identity.

In [3] it was shown that the bounded approximate identity in $A_{M}\left(F_{2}\right)$ is also bounded in $\|\cdot\|_{M}$. Moreover, if $G=\operatorname{SL}(2, \mathbb{R}), G=\operatorname{SO}(n, 1)$, or $G$ is any closed subgroup of any of these groups, then $A(G)$ has an approximate identity bounded in $\|\cdot\|_{M}$. Therefore we have:

Theorem 4. Let $G$ be $\operatorname{SL}(2, \mathbb{R}), \operatorname{SO}(n, 1)$, or $F_{n}$ for $n=2,3, \ldots$ Let $\mathscr{A}$ be either $A_{M}(g)$ or $A_{M_{0}}(G)$. Then every homomorphism for $A$ with finitedimensional range is continuous and every derivation from $\mathscr{A}$ into a Banach $\mathscr{A}$-bimodule is continuous.

Proof. This follows immediately from Theorem 2, Theorem 3, and [3, Theorem 3.7].

De Canniere and Haagerup had speculated about the existence of a bounded approximate identity in $A_{M}(G)$ and $A_{M_{0}}(G)$ for any locally compact group [3]. This would have provided a large new class of Banach spaces without discontinuous derivations, however, it has recently been shown that this is not the case (see [1]).

It would be of interest to know whether the above automatic continuity properties are characteristic of those groups for which either $A_{M}(G)$ or $A_{M_{0}}(G)$ has a bounded approximate identity.

\section{BIBLIOGRAPHY}

1. M. Cowling and U. Haagerup, Completely bounded multipliers of the Fourier algebra of a simple Lie group of real rank one, Invent. Math. 96 (1989), 507-549.

2. H. G. Dales and G. Willis, Cofinite ideals in Banach algebras and finite dimensional representations of group algebras, Proc. Conf. Automatic Continuity, Lecture Notes in Math., vol. 975, Springer-Verlag, New York, 1982.

3. J. De Canniere and U. Haagerup, Multipliers of the Fourier algebras of some simple Lie groups and their subgroups, Amer. J. Math. 107 (1985), 455-500.

4. P. Eymard, L'algèbre de Fourier d'un groupe localement compact, Bull. Soc. Math. France 92 (1964), 181-236.

5. A. Figa-Talamenca, A remark on multipliers of the Fourier algebra of the free group, Boll. Un. Mat. Ital, A (6) 16 (1979), 571-581. 
6. B. Forrest, Amenability and derivations of the Fourier algebra, Proc. Amer. Math. Soc. 104 (1988), 437-442.

7. __ Amenability and bounded approximate identities in ideals of $A(G)$, Illinois J. Math. 34 (1990), 1-25.

8. __ On amenability and some properties of $\operatorname{Ap}(G)$, preprint.

9. E. Hewitt and K. A. Ross, Abstract harmonic analysis, vol. 11, Springer-Verlag, New York, 1970.

10. N. P. Jewell, Continuity of module and higher derivations, Pacific J. Math. 68 (1977), 91-98.

11. A. T. Lau, Analysis on a class of Banach algebras with applications to harmonic analysis on locally compact groups and semigroups, Fund. Math. 118 (1983), 161-175.

12. V. Losert, Properties of the Fourier algebra that are equivalent to amenability, Proc. Amer. Math. Soc. 91 (1984), 347-354.

13. C. Nebbia, Multipliers and asymptotic behaviour of the Fourier algebra of nonamenable groups, Proc. Amer. Math. Soc. 84 (1982), 549-554.

Department of Pure Mathematics, University of Waterloo, Waterloo, Ontario CANADA 